\title{
Abgekürzt zitierte Werk- und Briefausgaben
}

Briefwechsel Bettina von Arnims

Hauptausgabe

GW 4

Bettine von Arnim: Werke und Briefe in vier Bänden. Bd. 4: Briefe. Hg. v. Heinz Härtl, Ulrike Landfester u. Sibylle von Steinsdorff. Frankfurt a.M.: Deutscher Klassiker Verlag, 2004 (= Bibliothek Deutscher Klassiker, Bd. 186).

Weitere Ausgaben

Bw Arnim 1

Bettine und Arnim. Briefe der Freundschaft und Liebe. 2 Bde. Bd. 1: 1806-1808; Bd. 2: 1808-1811. Hg., eingeführt u. kommentiert v. Otto Betz u. Veronika Straub. Frankfurt a.M.: Knecht, 1986, 1987.

Bw Arnim 2 Achim und Bettina in ibren Briefen. Briefwechsel von Achim von Arnim und Bettina Brentano. 2 Bde. Hg. v. Werner Vordtriede. Frankfurt a.M.: Suhrkamp, 1961. Taschenbuchausgabe: Frankfurt a. M.: Insel, 1988.

Bw Arnim 3 Achim von Arnim - Bettine Brentano verh. von Arnim. Briefwechsel. 3 Bde. Vollständig nach den Autographen hg. v. Renate Moering. Wiesbaden: Reichert, 2019.

Bw Döring Bettine von Arnim: Letzte Liebe. Das unbekannte Briefbuch. Hg. v. Wolfgang Bunzel. Berlin: Die Andere Bibliothek, 2019.

Bw Freimund Du bist mir Vater und Bruder und Sohn. Bettine von Arnims Briefwechsel mit ibrem Sohn Freimund. Hg. v. Wolfgang Bunzel u. Ulrike Landfester. Göttingen: Wallstein, 1999 (= Bettine von Arnims Briefwechsel mit ihren Söhnen, Bd. 1).

Bw Freyberg Der Briefwechsel zwischen Bettine Brentano und Max Prokop von Freyberg. Hg. u. komm. v. Sibylle von Steinsdorff. Berlin, New York: De Gruyter, 1972.

Bw Friedrich Wilhelm IV. „Die Welt umwälzen - denn darauf läufts hinaus". Der Briefwechsel zwischen Bettina von Arnim und Friedrich Wilhelm IV. 2 Bde. Hg. u. kommentiert v. Ursula Püschel, unter Mitarbeit v. Leonore Krenzlin. Bielefeld: Aisthesis, 2001.

Bw Friedmund In allem einverstanden mit Dir. Bettine von Arnims Brief- 
wechsel mit ihrem Sohn Friedmund. Hg. v. Wolfgang Bunzel u. Ulrike Landfester. Göttingen: Wallstein, 2001 (= Bettine von Arnims Briefwechsel mit ihren Söhnen, Bd. 3).

Bw Grimm Der Briefwechsel Bettine von Arnims mit den Brüdern Grimm 1838-1841. Hg. v. Hartwig Schultz. Frankfurt a. M.: Insel, 1985.

Bw Hössli Bettina von Arnim: „Ist Dir bange vor meiner Liebe?“ Briefe an Philipp Hössli, nebst dessen Gegenbriefen und Tagebuchnotizen. Hg. v. Kurt Wanner. Frankfurt a.M., Leipzig: Insel, 1996.

Bw Pückler Bettine von Arnim und Hermann von Pückler-Muskau: „Die Leidenschaft ist der Schlüssel zur Welt“. Briefwechsel 1832-1844. Hg. u. erläutert v. Enid Gajek u. Bernhard Gajek. Stuttgart: Cotta, 2001.

Bw Siegmund $\quad D a$ wir uns nun einmal nicht vertragen. Bettine von Arnims Briefwechsel mit ihrem Sohn Siegmund. Hg. v. Wolfgang Bunzel u. Ulrike Landfester. Göttingen: Wallstein, 2012 (= Bettine von Arnims Briefwechsel mit ihren Söhnen, Bd. 2).

Werkausgaben Bettina von Arnims

Hauptausgabe

GW 1

Bettine von Arnim: Werke und Briefe in vier Bänden. Bd. 1: Clemens Brentano’s Frühlingskranz / Die Günderode. Hg. v. Walter Schmitz u. Sibylle von Steinsdorff. Frankfurt a.M.: Deutscher Klassiker Verlag, 1986 (= Bibliothek Deutscher Klassiker, Bd. 12).

GW 2

Bettine von Arnim: Werke und Briefe in vier Bänden. Bd. 2: Goethe's Briefwechsel mit einem Kinde. Hg. von Walter Schmitz u. Sibylle von Steinsdorff. Frankfurt a.M.: Deutscher Klassiker Verlag, 1992 (= Bibliothek Deutscher Klassiker, Bd. 76).

GW 3 Bettine von Arnim: Werke und Briefe in vier Bänden. Bd. 3: Politische Schriften. Hg. v. Wolfgang Bunzel, Ulrike Landfester, Walter Schmitz und Sibylle von Steinsdorff. Frankfurt a.M.: Deutscher Klassiker Verlag, 1995 (= Bibliothek Deutscher Klassiker, Bd. 119).

Weitere Ausgaben

GW [Härtl] 1-2 Bettina von Arnim: Werke. 2 Bde. Im Auftrag der Nationalen Forschungs- und Gedenkstätten der klassischen 
deutschen Literatur in Weimar hg. v. Heinz Härtl. Bd. 1: Goethe's Briefwechsel mit einem Kinde. Berlin,Weimar: Aufbau, 1986. Bd. 2: Die Günderode / Clemens Brentano's Frühlingskranz. Berlin, Weimar: Aufbau, 1989.

GW [Konrad] 1-5 Bettina von Arnim: Werke und Briefe. 5 Bde. Hg. v. Gustav Konrad (Bd. 5 v. Joachim Müller). Frechen: Bartmann, 1959-1963.

GW [Oehlke] 1-7 Bettina von Arnim: Sämtliche Werke. 7 Bde. Hg. mit Benutzung ungedruckten Materials v. Waldemar Oehlke. Berlin: Propyläen, 1920-1922.

FBA 30 Clemens Brentano: Sämtliche Werke und Briefe. Historisch-kritische Ausgabe (= Frankfurter Brentano-Ausgabe). Bd. 30: Briefe II: „Clemens Brentano's Frühlingskranz" und handschriftlich überlieferte Briefe Brentanos an Bettine, 1800-1803. Hg. v. Lieselotte Kinskofer. Stuttgart: Kohlhammer, 1990.

Weitere Ausgaben mit Bezug zu Bettina von Arnim

Varnhagen 1

Varnhagen 2

Weitere Siglen

Bettine-Katalog

Jb BvA

Jb FDH
Aus dem Nachlaß Varnhagen's von Ense. Briefe von Stägemann, Metternich, Heine und Bettina von Arnim, nebst Briefen, Anmerkungen und Notizen von Varnhagen von Ense. Leipzig: Brockhaus, 1865. - Photomechanischer Nachdruck: Bern 1972.

Aus dem Nachlaß Varnhagen's von Ense. Tagebücher von K.A. Varnhagen von Ense. 14 Bde. Bd. 1-6: Leipzig, Brockhaus, 1861-1862; Bd. 7/8: Zürich: Meyer und Zeller, 1865; Bd. 9-14: Hamburg: Hoffmann und Campe, 1868-1870. - Photomechanischer Nachdruck: Bern 1972.

Christoph Perels (Hg.): „Herzhaft in die Dornen der Zeit greifen ...": Bettine von Arnim 1785-1859. Ausstellung Freies Deutsches Hochstift - Frankfurter GoetheMuseum. Frankfurt a.M.: Frankfurter Goethe-Museum, 1985.

Internationales Jahrbuch der Bettina-von-Arnim-Gesellschaft. Berlin: Saint-Albin [aktuell], $1987 \mathrm{ff}$. Jabrbuch des Freien Deutschen Hochstifts. Göttingen: Wallstein [aktuell], $1902 \mathrm{ff}$. 